\title{
Atomic Resolution Electron Diffraction Imaging of Metallic Nanoclusters
}

\author{
W.J. Huang, B. Jiang and J.M. Zuo
}

Department of Materials Science and Engineering, Ferdrick Seitz Materials Research Laboratory, University of Illinois at Urbana-Champaign, Urbana, IL, 61801, United States.

Imaging non-crystalline objects at atomic-resolution, such as macromolecules or individual nanoclusters, remains as a significant challenge for electron microscopy. With the development of $\mathrm{C}_{\mathrm{s}}$ correctors, the image resolution of electron microscopes is significantly improved [1]. However, information that can be obtained from electron images is still limited by loss of contrast due to electron energy spread and limited coherence [2]. In comparison, electron diffraction imaging, a recently developed technique, takes advantage of the high resolution information available in oversampled diffraction patterns, and combines with iterative algorithms to retrieve the missing phases and reconstruct the object images beyond the aberration limits [3]. The resolution is only limited by diffraction intensity. The recent work by Wu and Spence shows one such example for a $13 \mathrm{~nm}$ diameter Au particle [4]. Here we explore the possibility of applying the diffraction imaging technique to image the structures of small $(5 \mathrm{~nm}$ or smaller) metallic nanoclusters, for which improving image resolution and contrast and limiting electron beam induced structural changes are all critical issues

To demonstrate the feasibility of electron diffraction imaging for small metal particles, a computer diffraction experiment is conducted on a $4 \mathrm{~nm}$-sized Au icosahedral nanocluster (Fig. 1). The dynamical diffraction pattern (Fig. 2) is generated by using the multislice method [5]. Phase retrieval algorithms are used to solve the phase problem for the diffraction data. Specifically, we used a combination of a modified error reduction [6], hybrid-input-output [6] and charge flipping algorithms [4]. The phase retrieval algorithm iterates between the object and Fourier domains, where the constraints are applied. With dynamical scattering, the electron wave function at the exit plane is complex. One issue, thus associated with electron diffraction, is to what limit that the real object constraint, which is equivalent to the kinematical approximation, can be applied to the exit wave function reconstruction. To address this issue, a kinematical diffraction pattern of the Au nanocluster is also generated and compared with the dynamical diffraction pattern obtained by the multislice method. For the kinematical diffraction pattern, real object constraint is applied for the phase retrieval; while for the dynamical diffraction pattern, phase retrieval is performed with and without the real object constraint for comparison. Fig. 3 shows the reconstructed object using the real object constraint from the dynamical diffraction pattern. The reconstructed image is closer to the intensity of exit wave function than the projected potential (Fig. 1). Solving the phase problem by iterative algorithms requires sampling the diffraction pattern at interval finer than the Nyquist frequency [3], which is equivalent to having a finite support for the object. Estimating a tight enough support was found to be crucial to the reconstruction, therefore an algorithm which dynamically drives a starting support to approach the actual physical support (Fig. 4) is developed and used in our phase retrieval program. Finally, noise, which ultimately determine the achievable resolution, is simulated to examine the applicability of this method to real data. 
In conclusion, our computer diffraction experiment shows that dynamical diffraction patterns for small multiply- twinned Au particles can be iteratively phased by applying real object constraint while maintaining atomic resolution and avoiding significant constraint-induced errors in reconstructed images. The dynamically estimated support is essential to achieve sub-angstrom resolution for reconstructed objects.

[1] P. D. Nellist et al., Science, Vol.305, 1741, 2004

[2] M.J. Hytch \& W.M. Stobbs, Ultramicroscopy, Vol.53, 191, 1994

[3] J.M. Zuo et al., Science, Vol.300, 1419, 2003

[4] J.S. Wu and J.C.H Spence, Nature Materials, Vol.4, 912, 2005

[5] J.M. Cowley and A.F. Moodie, Acta Cryst., Vol.10, 609, 1957

[6] J.R. Fienup, Applied Optics, Vol.21, 2758, 1982

[7] This work is supported by NSF DMR-0449790 (WJH, JMZ) and DOE BES DE-FG02$01 \mathrm{ER} 45923$ (BJ, JMZ).
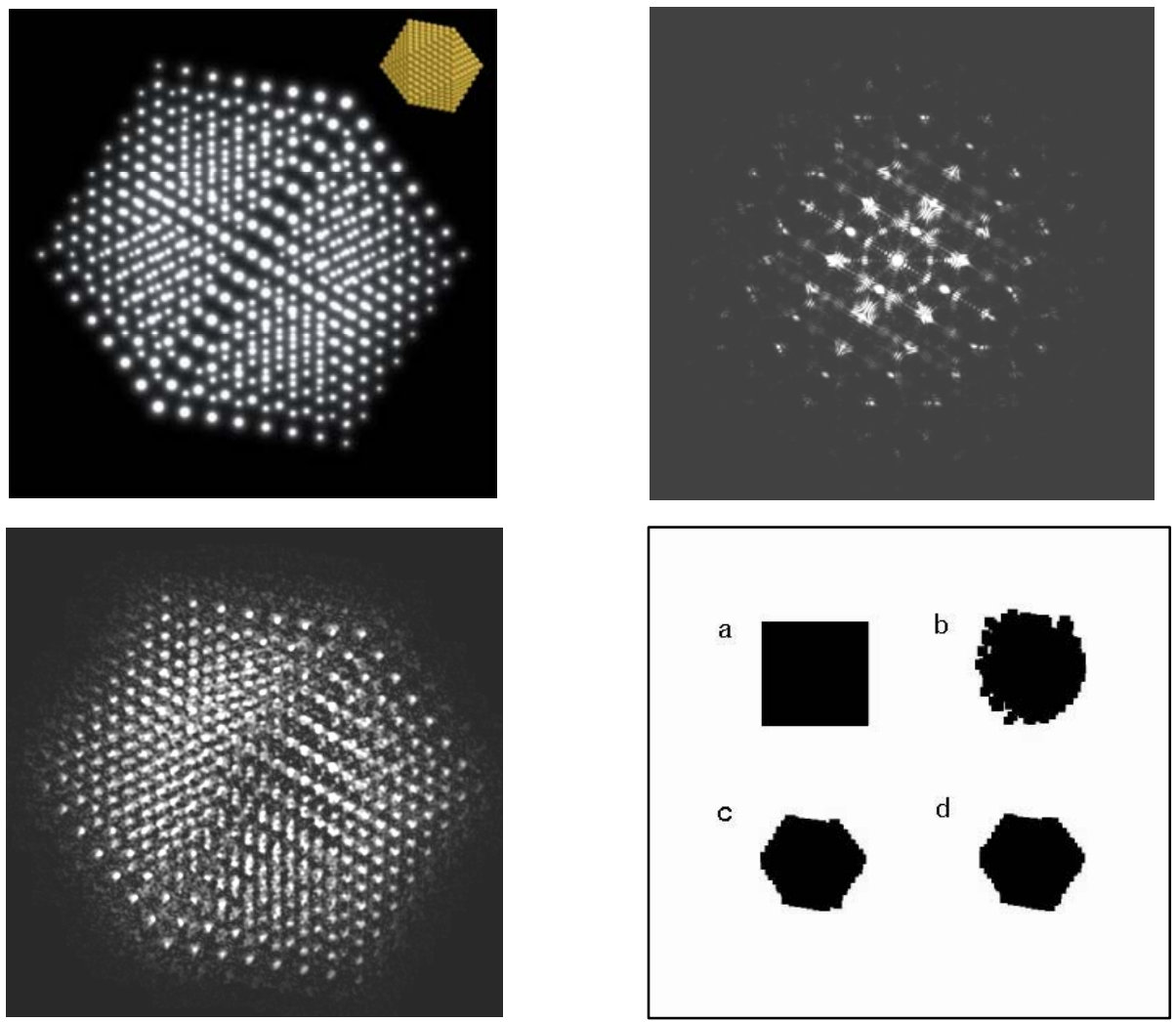

Fig.1 (upper left): The projected potential of a $4 \mathrm{~nm}$ icosahedral Au nanocluster along one of the 2-fold axes; the structural model is shown in the upper right corner.

Fig.2 (upper right): The simulated dynamical electron diffraction pattern of the Au nanocluster shown in fig. 1 , by the multislice method.

Fig.3 (bottom left): The reconstructed image of the Au nanocluster from the diffraction pattern shown in fig.2. The reconstruction employs a combination of the error reduction, hybrid-inputoutput and charge flipping algorithms. The finite support of the object is estimated dynamically during each iteration.

Fig.4 (bottom right): Evolution of the dynamic support of the object: object support at the 0th (a), 5th (b), 15th (c) and 25th (d) iteration. 